\title{
Crystallographic Orientation Control of 316L Austenitic Stainless Steel via Selective Laser Melting
}

\author{
Takuya ISHIMOTO, ${ }^{1,2)}$ Siqi WU, ${ }^{1)}$ Yukinobu ITO, ${ }^{1)}$ Shi-Hai SUN,${ }^{1,2)}$ Hiroki AMANO ${ }^{1,3)}$ and Takayoshi NAKANO ${ }^{1,2) *}$ \\ 1) Division of Materials and Manufacturing Science, Graduate School of Engineering, Osaka University, 2-1 Yamada-Oka, \\ Suita, Osaka, 565-0871 Japan. \\ 2) Anisotropic Design \& Additive Manufacturing Research Center, Osaka University, 2-1 Yamada-Oka, Suita, Osaka, 565-0871 \\ Japan. \\ 3) TAIYO NIPPON SANSO Corporation, 1-3-26 Koyama, Shinagawa-ku, Tokyo, 142-8558 Japan.
}

(Received on November 16, 2019; accepted on January 23, 2020; J-STAGE Advance published date: March 11, 2020)

\begin{abstract}
In recent years, additive manufacturing has attracted attention as a technology that enables control of the crystallographic texture of metallic materials. We achieved successful control of the crystallographic texture of $316 \mathrm{~L}$ austenitic stainless steel using selective laser melting (SLM). Three distinguished textures were achieved by changing the laser scan speed, namely: the single crystalline-like texture with $\{001\}$ orientation in the build direction, the crystallographic lamellar texture in which two kinds of grains with $\{011\}$ and $\{001\}$ orientations in the build direction are alternately stacked, and polycrystalline with relatively random orientation. The melt pool shape and the solidification behavior (thermal gradient and migration velocity of solid/liquid interface) in a melt pool could be important controlling factors for the evolution of the crystallographic texture under the SLM process.
\end{abstract}

KEY WORDS: crystallographic texture; metal additive manufacturing (AM); selective laser melting (SLM); melt pool; anisotropy.

\section{Introduction}

Metal additive manufacturing (AM) has attracted the most attention in recent years among various metal processing technologies. Conventionally, metal AM has been characterized by its flexible shaping ability and relatively high processing speed. Taking advantage of such features of AM, metal AM technology has been applied to the fabrication of products with hollow structures, three-dimensional complex porous bodies, and tailor-made products, among others. ${ }^{1,2)}$ Meanwhile, in recent studies, metal AM has emerged as a methodology for controlling the crystallographic texture of metallic materials ${ }^{3-5)}$ in addition to controlling the shape of products. Crystallographic texture, which is an important material parameter for metallic materials, is a major factor that affects the functionality of a product, independent of shape parameters.

The mechanical properties of metallic materials are the most significant factor controlled by the crystallographic texture. Highly texturized materials with preferential crystallographic orientations can feature mechanical anisotropies, such as Young's modulus, ${ }^{6,7)}$ yield stress and elongation, ${ }^{8)}$ fatigue resistance, ${ }^{9)}$ and creep resistance. ${ }^{10)}$ The single crystalline form, in which the anisotropy based on the atomic arrangement is manifested to the limit, exhibits

\footnotetext{
* Corresponding author: E-mail: nakano@mat.eng.osaka-u.ac.jp DOI: https://doi.org/10.2355/isijinternational.ISIJINT-2019-744
}

a maximum anisotropy in Young's modulus among many metal materials. For example, in a $\beta$-type titanium alloy with a bcc structure, the minimum value of Young's modulus is shown in the $<001>$ orientation, while the maximum value is in the $<111>$ orientation by forming a single crystalline. ${ }^{6,7)}$ These values are remarkably lower or higher than those exhibited by a polycrystalline body, respectively. This indicates the advantage in producing structural parts, being that the degree of freedom in selecting the Young's modulus is expanded due to the selection of polycrystalline and single crystalline, although a single material is used. Meanwhile, the crystallographic state has a significant effect on the yield and fatigue behaviors based on the influence on the dislocation mobility, which is governed by the geometry of the atomic arrangement and grain boundaries that inevitably exist at the interfaces of crystal grains with different atomic arrangements. Thus, the control of the crystallographic texture from single crystalline to polycrystalline by AM is considered to be very beneficial as a means to expand the mechanical functionality of the metallic products to be selected.

316L stainless steel (316L SS) is a fully austenitic stainless steel known for its excellent corrosion resistance (e.g., resistance to pitting corrosion) and mechanical properties. Furthermore, $316 \mathrm{~L}$ SS is a common material used in a variety of industries, such as chemical, petrochemical, marine, and medical. Therefore, the crystallographic texture control of $316 \mathrm{~L} \mathrm{SS}$ may result in products of increased function- 
alities in a wide range of applications. The purpose of this study was to control the crystallographic texture of 316L SS via selective laser melting (SLM), a powder bed fusion-type metal AM using a laser as the heat source.

\section{Materials and Methods}

Gas atomized 316L SS powder (Fig. 1) was obtained from EOS GmbH (Germany). The nominal composition of the powder was $18 \mathrm{Cr}-14 \mathrm{Ni}-2.5 \mathrm{Mo}-0.03 \mathrm{C}$ (wt.\%), and the powder size was under $53 \mu \mathrm{m}$. SLM fabrication was conducted using an EOS M290 printer equipped with a Yb-fiber laser by the "X-scan strategy," i.e., the laser beam was scanned bidirectionally along the $x$-axis without rotation (Fig. 2(a)). The X-scan strategy has been clarified to be beneficial in producing a single crystalline-like texture ${ }^{3,4,11)}$ because of the fixed laser scanning direction and the resultant homogenous thermal flux in each melt pool. In this study, the build direction was defined as the $z$-direction, and the direction orthogonal to the $x$ - and $z$-directions was defined as the $y$-direction (Fig. 2(a)). By changing the laser scan speed $(v)$, the energy density $(E)$ was controlled, while the laser power $(P)$ was kept constant. The hatching distance (h) and layered thickness $(t)$ were set to $80 \mu \mathrm{m}$ and $40 \mu \mathrm{m}$, respectively. Three laser scan speeds, low, medium, and high, were used; the faster laser scan speed corresponds to a lower energy density as $E=P /(v h t)$. Products of $10 \mathrm{~mm} \times$ $10 \mathrm{~mm} \times 10 \mathrm{~mm}$ were fabricated (Fig. 2(b)), thus, laser

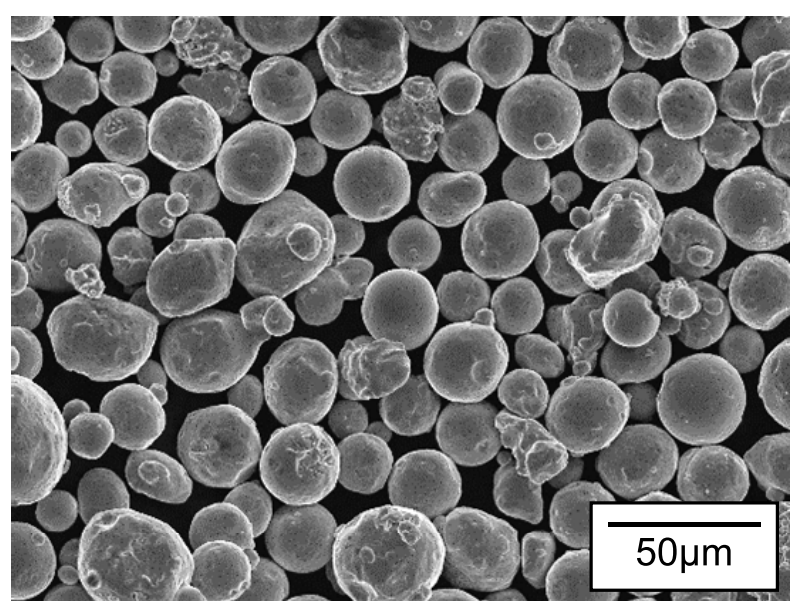

Fig. 1. SEM micrograph of 361L SS powder used. was scanned 125 passes in each layer for 250 layers. The specimens fabricated at low, medium, and high speed are referred to as Specimen_A, Specimen_B, and Specimen_C, respectively.

The samples were cut from a stainless-steel base-plate by electric discharge machining. All the analyses were performed in the as-built specimen without any heat treatment. The densities were measured using the Archimedes method. The specimens were cut along the $y z$-plane, mechanically abraded, and chemically etched in a solution comprising $21 \%$ hydrofluoric acid, 29\% nitric acid, and 50\% water. The melt pool shape and microstructures were observed by optical microscopy (OM; BX60, Olympus, Japan) and field-emission scanning electron microscopy (FE-SEM; JEOL JIB-4610F, Japan). The crystallographic texture was examined by electron backscatter diffraction (EBSD). In addition, the Kikuchi diffraction pattern was detected by a NordlysMax ${ }^{3}$ system (Oxford Instruments, UK) and analyzed using AZtecHKL software (Oxford Instruments, UK). To quantitatively characterize the evolved texture, the degree of orientations $(p)$ of $\{001\}$ and $\{011\}$ with respect to the build direction were calculated based on the Euler angles obtained by EBSD as follows:

$$
p_{\{h k l\}}=\left\langle\cos ^{2} \alpha_{\{h k l\}}\right\rangle .
$$

Here, $\alpha_{\{h k l\}}$ shows the angle of $\{001\}$ or $\{011\}$ from the build direction derived from Euler angles at each analysis point in EBSD, and the angle brackets represent the average at all analyzed points. Considering the multiplicity of the crystal planes, the orientation degrees for the random orientations of $\{001\}$ and $\{011\}$ are 0.70 and 0.84 , respectively. A perfect orientation leads to $p=1$.

The quantitative data for the melt pool shape are given as the mean \pm standard deviation (SD). Comparisons between the means were statistically performed using one-way analysis of variance (ANOVA) and post hoc Tukey HSD tests (IBM SPSS Statistics 25; SPSS Japan, Japan). A value of $P<0.05$ was considered statistically significant.

\section{Results and Discussion}

The $10 \mathrm{~mm} \times 10 \mathrm{~mm} \times 10 \mathrm{~mm}$ specimens were successfully fabricated using SLM by appropriately tuning the process parameters. The absolute densities of Specimen_A, B,
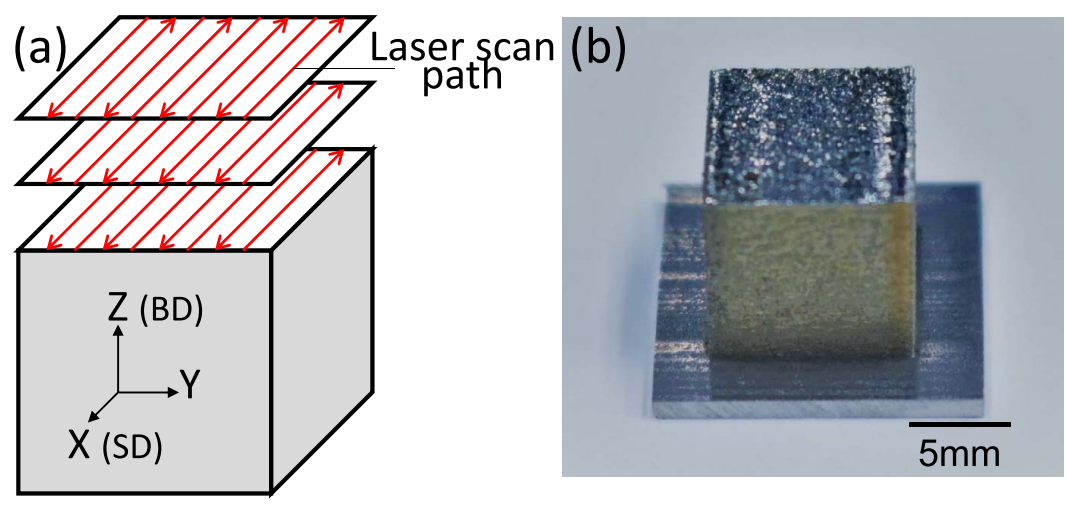

Fig. 2. (a) Schematic of the "X-scan strategy" and (b) typical appearance of the product fabricated via SLM. (Online version in color.) 
and $\mathrm{C}$ were measured by the Archimedes' method as 7.85 , 7.95 , and $7.97 \mathrm{~g} / \mathrm{cm}^{3}$, corresponding to relative densities of $98.4 \%, 99.7 \%$, and $99.9 \%$, respectively. The cross-sectional OM pictures are shown in Fig. 3. Many spherical pores formed in Specimen_A (Fig. 3(a)), which was fabricated under a low laser scanning speed (high energy density). As described later, the melt pool of Specimen_A formed in the keyhole mode, and the pores were generated besides the formation of the keyhole. ${ }^{12)}$ The lack-of-fusion-type irregular pores were not observed in any specimen, indicating that the input heat energies through laser irradiation were adequately strong.

Figure 4 shows the crystallographic textures that evolved in the specimens. The inverse pole figure (IPF) maps (Figs. 4(a)-4(i)) illustrate the crystallographic textures of the specimens projected in the $x-, y$-, and $z$-directions, created from the EBSD analysis in the $y-z$ cross-section. The corresponding $\{001\}$ pole figures are represented in Figs. 4(j) 4(1). Importantly, the crystallographic texture and orientation varied significantly depending on the laser scan speed. This transition of the texture was not necessarily consistent with the scan speed. Specimen_A, fabricated at low speed, showed a cube texture with $\{001\}$ preferentially oriented in all of the scan $(x$-) directions, the $y$-direction, and the build (z-) direction (Figs. 4(a), 4(d), 4(g), 4(j)). Meanwhile, although Specimen_B, fabricated at medium scan speed,

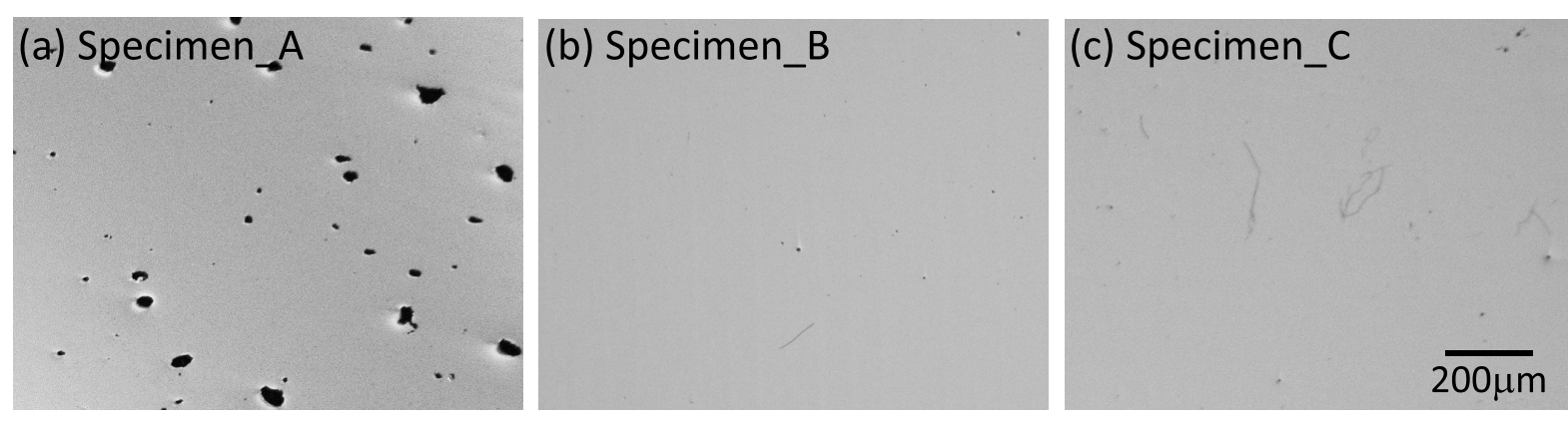

Fig. 3. SEM micrograph of the $y-z$ cross-section.
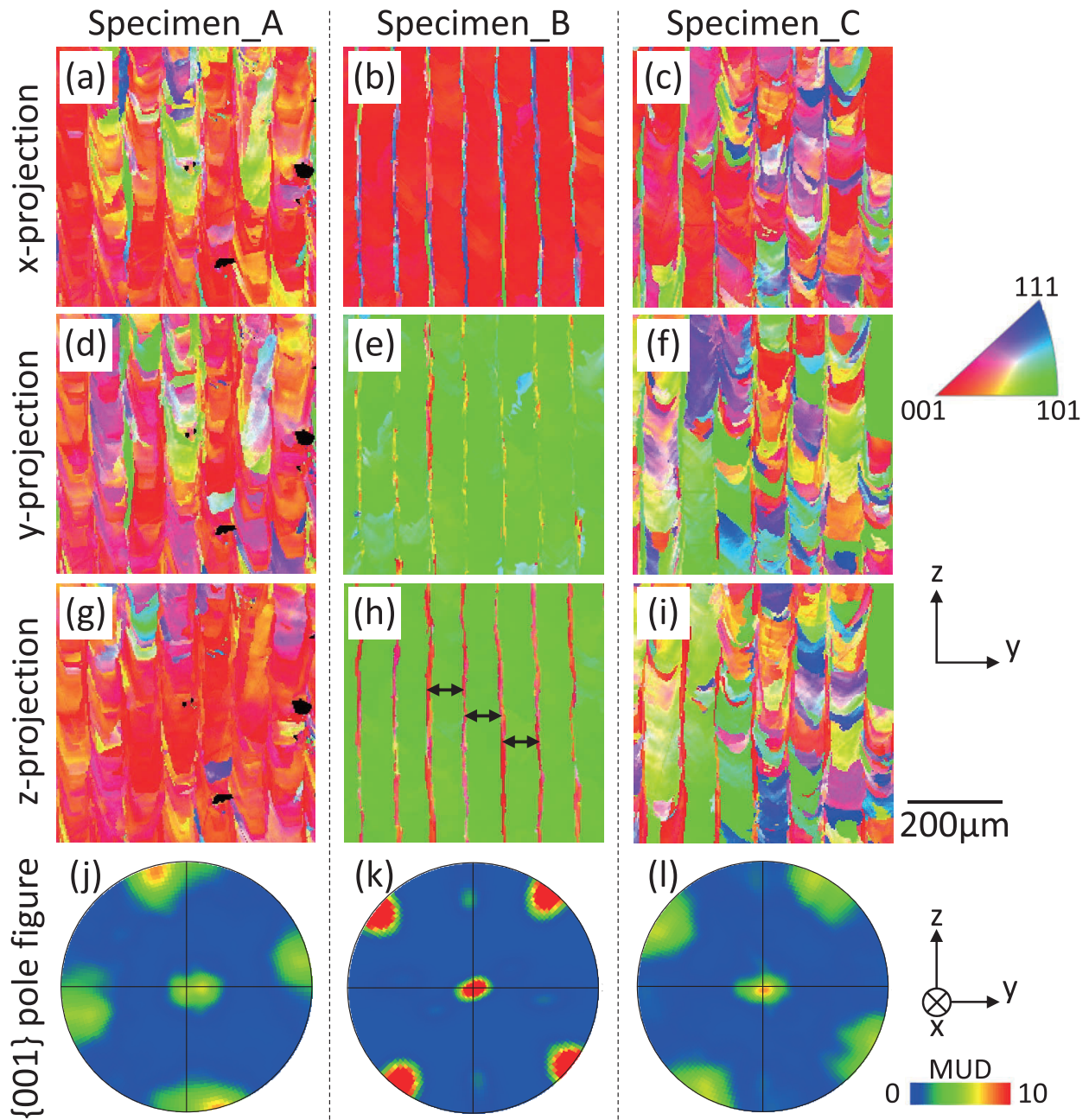

Fig. 4. (a-i) IPF maps taken in the $y-z$ cross-section and $(\mathrm{j}-1)\{001\}$ pole figures. Double-headed arrows in (h) indicate the length of one cycle of periodic CLM structure. 
mostly exhibited a texture with $\{011\}$ oriented in the $z$-and $y$-directions and $\{001\}$ oriented in the $x$-direction, as shown in Figs. 4(b), 4(e), 4(h), 4(k), a thin layer with $\{001\}$ orientation in the $z$-direction was periodically included, as clearly seen in Fig. 4(h). This unique microstructure is referred to as "crystallographic lamellar microstructure (CLM)". ${ }^{13)}$ Its periodicity coincides with the laser hatching distance (80 $\mu \mathrm{m})$ and is thus very fine, which can be formed by SLM but not by other processing techniques. In Specimen_C, the orientation was remarkably randomized while it maintained to some extent the texture seen in Specimen_B. This texture was quantified as the orientation degrees of $\{001\}$ and $\{011\}$ along the build direction, as shown in Fig. 5. It is clear that the microstructure of Specimen_B is highly single crystalline-like, indicated by the quite high degree of orientation for $\{011\}$ close to 1 . A slight drop from 1 is due to the formation of the abovementioned thin layer with preferential $\{001\}$ orientation in the $z$-direction. According to the pole figures with a standardized intensity (Figs. 4(j), 4(1)), the strengths of the crystallographic orientations in Specimen_A and C appear to be similar. Based on the calculated orientation degree (Fig. 5), however, it can be understood that Specimen_A has a more prominent texture than Specimen_C, which exhibits orientation degrees close to those of random orientations (disordered orientation).

Both Specimen_A and B have a strong tendency to inherit the crystal orientation in the build direction over multiple layers, forming a columnar microstructure. In SLM with track-by-track and layer-by-layer melting, the underlying and adjacent parts previously deposited are partly remelted. Thus, an epitaxial crystal growth from the previously solidified parts occurs. In Specimen_C, in contrast, new grains with different crystallographic orientations tend to form while showing the characteristics of epitaxial growth, resulting in polycrystalline structure with relatively random orientation. Explanation of such a transition of the microstructure has been attempted according to the theories of alloy solidification; ${ }^{14,15)}$ the transition might be related to the solid/liquid interface morphology, which is largely affected by constitutional supercooling. For a certain alloy, the degree of constitutional supercooling is determined by the ratio of the thermal gradient $(G)$ and the migration

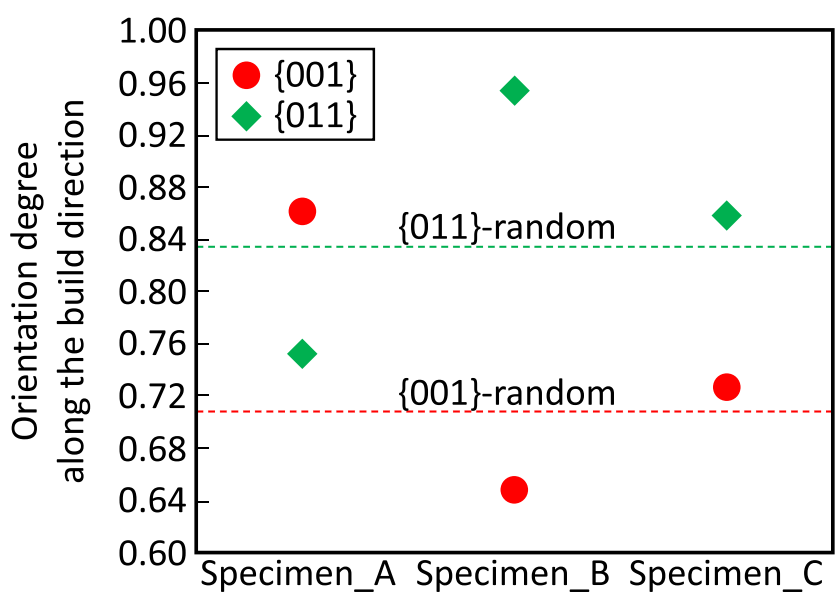

Fig. 5. Orientation degrees of $\{001\}$ and $\{011\}$ along the build direction. (Online version in color.) velocity of the solid/liquid interface $(R) .{ }^{16)}$ The increased $G / R$ leads to a stable planar or cellular interface and the resultant columnar microstructure, while the decreased $G / R$ leads to an equiaxed microstructure due to significant constitutional supercooling. Constitutional supercooling, and thus the solid/liquid interface morphology, depend on the material composition, therefore, unless the transition point in $316 \mathrm{~L}$ SS and the actual $G$ and $R$ experienced in this study are quantitatively determined, the difference in the microstructure observed here cannot be fully explained. However, it is important to discuss the qualitative trends of the microstructural change. According to numerical simulations, $R$ increases as the scan speed increases ${ }^{17)}$ or the input energy decreases. $\left.{ }^{18}\right)$ This would qualitatively explain polycrystallization in Specimen_C fabricated with the high laser scan speed.

Another contributory factor for the evolution of the crystallographic texture might be the migration direction of the solid/liquid interface, which is closely related to that of the thermal gradient within a melt pool. To understand the relationship between the microstructure developed in the melt pool and the texture, an SEM micrograph and IPF map were taken in the same region. Figure 6 shows the SEM micrographs and IPF maps overlaid with the traces of the melt pool edges observed in the $y-z$ cross-section. Within a layer, fabrication was proceeded right to left as it was clear that a melt pool was partially overwrote (remelted) by the neighboring left track. In Specimen_A and B, the crystallographic orientations were almost identical across the melt pool edge (Figs. 6(a), 6(b)), indicating that epitaxial growth occurred at the local solid/liquid interface. Furthermore, the cellular microstructure can be observed throughout the cross section. The elongated direction of the cellular microstructure seen in Specimen_A and B almost corresponds to $<001>$, which is an easy growth direction for metals with cubic structure. Interestingly, the cell elongation directions in the melt pool varied between Specimen_A and B, as the crystal orientations were significantly different. In the specimen fabricated with high scan speed (Specimen_A), the $<001>$-oriented cells bidirectionally developed along the build and horizontal (y-) directions (red arrows) to form a cube texture with $<001>$ parallel to the $x$-, $y$-, and $z$-directions. In the specimen fabricated with the medium scan speed (Specimen_B), cells developed along an incline of approximately $\pm 45^{\circ}$ with respect to the build direction (green arrows), accompanied by elongated cells parallel to the build direction (red arrows) at the center of the melt-pools, to form CLM, in which two kinds of layers with different orientations were alternately stacked. In the X-scan strategy, $\pm 45^{\circ}$ cell growth and the corresponding single crystalline-like texture with $\{011\}$ orientation in the build direction generally occurred as was previously well-documented. ${ }^{3,4)}$ The formation of CLM was achieved by appropriate modification of the laser condition based on the single-crystalline formation. ${ }^{13)}$ However, the single crystalline-like texture with $\{001\}$ orientation in the build direction is reported for the first time under the X-scan strategy in SLM.

To discuss the cause of such cell growth, the top layer of the built product was observed to examine the shape of the melt pool without the influence of remelting and repetitive heating, ${ }^{3)}$ which are distinctive features of layer-by-layer 

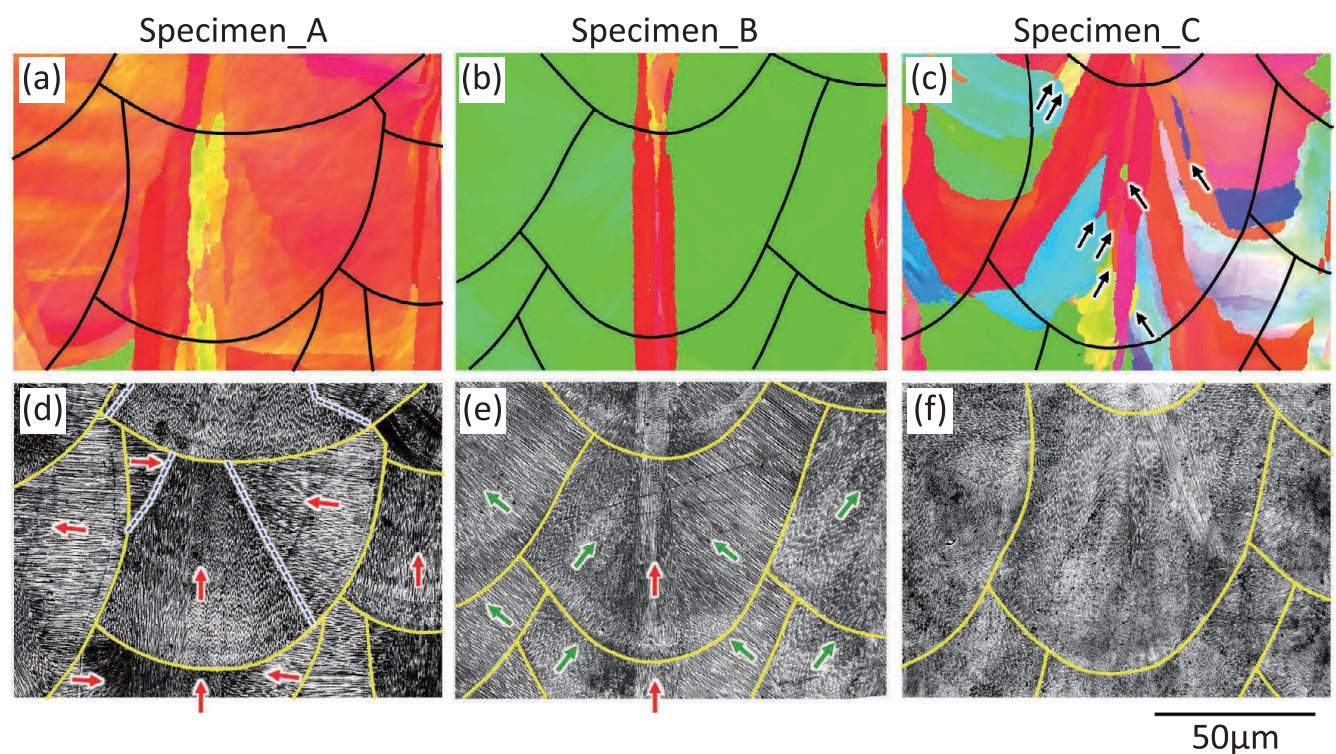

Fig. 6. (a-c) IPF maps and (d-f) corresponding SEM images showing the cellular microstructure taken on the $y$-z plane Melt pool cross-sections are partially observed owing to the layer-by-layer fabrication, and the melt pool edges are highlighted. The arrows in (c) indicate nucleated grains without inheriting crystallographic orientation, those in $(\mathrm{d}, \mathrm{e})$ indicate the direction of cell elongation (green: $\pm 45^{\circ}$-oriented cells; red: horizontally and vertically oriented cells), and the broken lines in (d) indicate the boundaries of the areas where the horizontally and vertically oriented cells were observed.
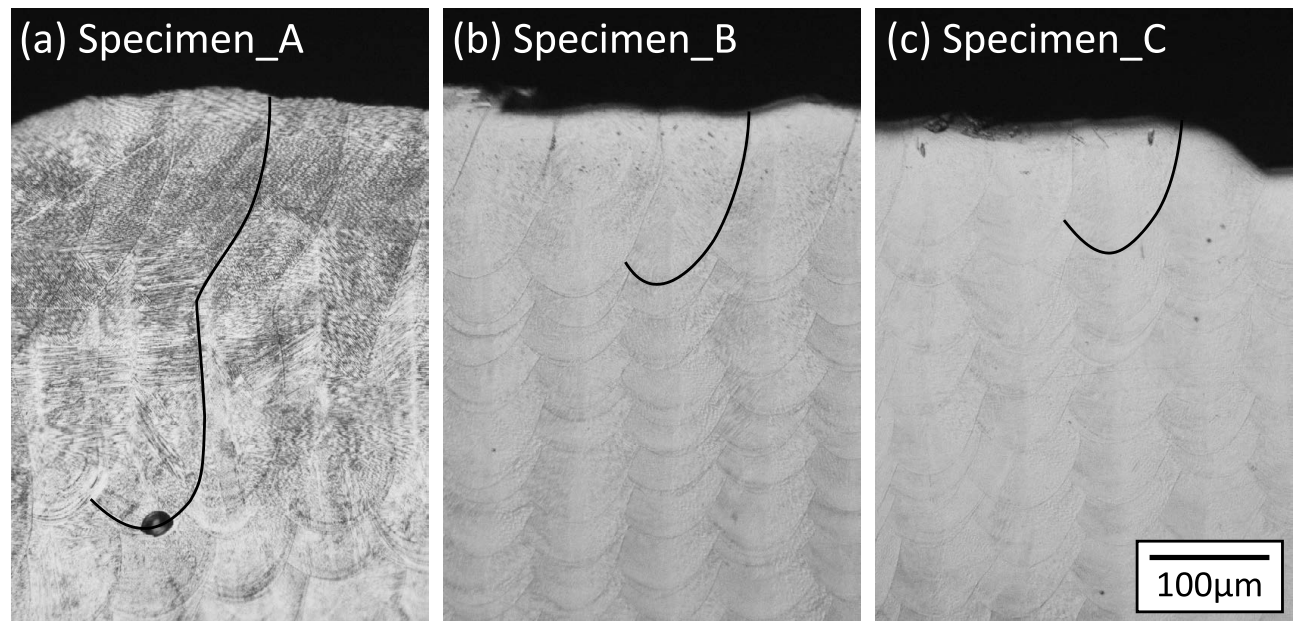

Fig. 7. OM images of the top layer of the built products showing full-depth of melt pool cross-sections.

fabrication. ${ }^{19)}$ Figure 7 shows the OM photographs of the $y-z$ plane in which cross-sections of the melt pool are observed. The melt pool edges are clearly seen. Figure 8 represents quantification of the melt pool size (width and depth). The width was determined as twice the half-width because one side was erased by the next track. Ten melt pools were randomly selected and used for the measurement. The slow speed, i.e., high input energy, significantly expanded the width and depth of the melt pool; however, the expansion of the depth was more prominent, resulting in a deep keyhole-type melt pool with a larger depth/width ratio in Specimen_A. The melt pool with a smaller depth/ width ratio, generated in Specimen_B, indicates conduction mode melting. It has been argued that the shape of the melt pool controls the crystallographic texture formation ${ }^{13,20)}$ because the melt pool shape reflects the temperature distribution; thus, affects the directions of the thermal gradient and solid/liquid interface migration. In the conduction

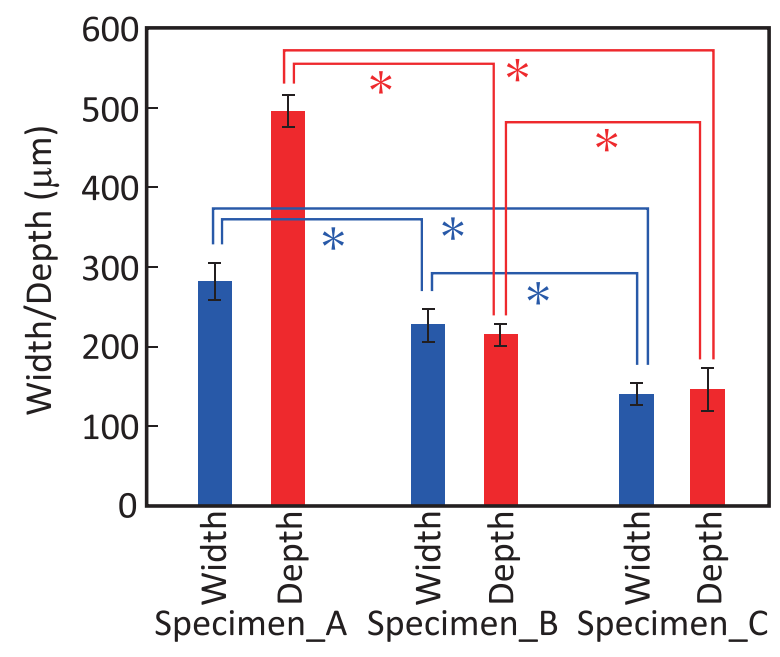

Fig. 8. Width and depth of the melt pools. *: $P<0.05$ by Tukey HSD tests. 
mode, the curvature of the melt pool bottom decreases during solidification, and the migration of the solid/liquid interface is frequently toward the build direction as solidification proceeds. ${ }^{21)}$ The directions of the thermal gradient and solid/liquid interface migration tend to be parallel to the build direction, which is preferable for the evolution of columnar cells with $<001>$ orientation along the build direction. When the vertically grown columnar cells are formed, the orientation is easily inherited through epitaxial growth. ${ }^{13)}$ Regarding the formation of the single-crystalline like texture with $\{001\}$ orientation in the build direction (Specimen_A), the melt pool sidewall that towers almost vertically and the relatively small curvature of the melt pool bottom are considered to be involved in the mechanism. Lateral migration of the solid/liquid interface at the melt pool sidewall and upward migration of the solid/liquid interface at the bottom take place and struggle with each other in the lower half of the deep keyhole-type melt pool, leading to bidirectional cell growth in the $y$ - and $z$-directions. Accordingly, the region in which the vertically grown cells are observed becomes narrower as it goes upward in a melt pool. An in-situ analysis or numerical simulation of the solidification behavior in the melt pool is necessary for a more advanced discussion of the mechanism underlying the texture formation in SLM.

There are many reports on the SLM fabrication of 316L $\mathrm{SS} ;{ }^{22)}$ however, to the best of the authors' knowledge, there are no reports of the creation of distinguished different textures. We have realized crystallographic texture control over a wide range: a single crystalline-like texture with $\{001\}$ orientation in the build direction, that with $\{011\}$ orientation in the build direction, ${ }^{13)}$ a CLM texture in which two kinds of grains with different orientations were alternately stacked in a periodicity of $80 \mu \mathrm{m}$, and polycrystalline. Texture control over a wide range enables the adjustment of the physical properties to those desired by selecting the type of texture and orientation, thereby expanding the range of utilization of the 316L SS alloy. Moreover, this strategy for texture control can be applicable to many other metallic materials. It should be noted that for different product sizes, the optimal laser conditions for obtaining such textures will vary, because temperature distribution is largely affected by the product size.

The merits of single crystallization of $316 \mathrm{~L} \mathrm{SS}$, for example, would apply to orthopedic implants. 316L SS is frequently employed for temporary devices in orthopedic surgery, such as bone plates, because of its favorable combination of mechanical properties, corrosion resistance, acceptable biocompatibility, and cost effectiveness compared with other metallic biomaterials. ${ }^{23)}$ However, the use of stiff metal implants induces stress shielding, in which stresses placed on the bone are supported by the stiff metal implant, leading to insufficient physiological loading on the bone. ${ }^{24,25)}$ Sound loading plays an important role not only in the maintenance of bone mass but also in that of bone qual$i$ ity, ${ }^{26,27)}$ therefore, the stress shielding should be avoided. ${ }^{28,29)}$ Polycrystalline-form 316L SS shows the Young's modulus of $\sim 200 \mathrm{GPa}^{30)}$ which is much higher than the modulus of bone $(\sim 30 \mathrm{GPa})$. Meanwhile, single-crystalline $316 \mathrm{~L}$ SS shows anisotropy in the Young's modulus; the lowest modulus was reported to be below $100 \mathrm{GPa}$ along <001>.31)
This value is comparable to or lower than the modulus of the Ti-6Al-4V alloy, which is most frequently used for orthopedic implant material. Single-crystalline 316L SS can mitigate stress shielding compared to the conventional polycrystalline form, and is expected to have a performance equivalent to that of the Ti-6Al-4V alloy from the viewpoint of stress shielding suppression. In addition, SLM-fabricated 316L SS products show significantly improved corrosion resistance; ${ }^{13)}$ hence, SLM will improve the in vivo performance of 316L SS as biomedical implants.

In general, the yield stress of single crystalline is low owing to the absence of grain boundaries that hinder dislocation movement. The products fabricated via SLM, however, can exhibit superior strength compared to counterparts with the same chemical compositions, made by a conventional method. ${ }^{32)}$ The superior strength is believed to mainly originate from the presence of a high population of dislocation densities in the as-fabricated products as a result of the presence of residual stress, and the cell wall. ${ }^{33)}$ Furthermore, the yield stress can be increased by forming CLM, that is, by artificially introducing grain boundaries. ${ }^{13)}$

SLM not only provides products with excellent material properties, but also enables control of the crystallographic texture formation and the resulting mechanical properties; therefore, it is a promising manufacturing technology for creating highly functional materials in the near future.

\section{Conclusion}

In this study, we successfully controlled the crystallographic texture of 316L SS via SLM. The single crystallinelike texture with $\{001\}$ orientation in the build direction, the CLM texture in which two kinds of grains with $\{011\}$ and $\{001\}$ orientations in the build direction were alternately stacked, and the polycrystalline with relatively random orientation were achieved under the X-scan strategy by changing the laser scan speed (or input energy density). The potential of SLM in controlling the microstructure and subsequent functionality was shown.

\section{Acknowledgements}

This work was supported by the Grants-in-Aid for Scientific Research (JP18H05254, JP17H06224, JP19H00827) from the Japan Society for the Promotion of Science (JSPS). This work was also partly supported by the CrossMinisterial Strategic Innovation Promotion Program (SIP), "Materials Integration for Revolutionary Design System of Structural Materials" from the Japan Science and Technology Agency (JST) and "Innovative Design/Manufacturing Technologies" from the New Energy and Industrial Technology Development Organization (NEDO), and the 26th (Grantee: T. Nakano) and 27th (Grantee: T. Ishimoto) ISIJ Research Promotion Grant from the Iron and Steel Institute of Japan (ISIJ).

\section{REFERENCES}

1) J. Plocher and A. Panesar: Mater. Des., 183 (2019), 108164. https:// doi.org/10.1016/j.matdes.2019.108164

2) T. Nakano, W. Fujitani, T. Ishimoto, J.-W. Lee, N. Ikeo, H. Fukuda and K. Kuramoto: ISIJ Int., 51 (2011), 262. https://doi.org/10.2355/ isijinternational.51.262 
3) T. Ishimoto, K. Hagihara, K. Hisamoto, S.-H. Sun and T. Nakano: Scr. Mater., 132 (2017), 34. https://doi.org/10.1016/j. scriptamat.2016.12.038

4) S.-H. Sun, K. Hagihara and T. Nakano: Mater. Des., 140 (2018), 307. http://doi.org/10.1016/j.matdes.2017.11.060

5) T. Nagase, T. Hori, M. Todai, S.-H. Sun and T. Nakano: Mater. Des., 173 (2019), 107771. https://doi.org/10.1016/j.matdes.2019.107771

6) S.-H. Lee, M. Todai, M. Tane, K. Hagihara, H. Nakajima and T. Nakano: J. Mech. Behav. Biomed. Mater., 14 (2012), 48. https://doi. org/10.1016/j.jmbbm.2012.05.005

7) M. Tane, S. Akita, T. Nakano, K. Hagihara, Y. Umakoshi, M. Niinomi and H. Nakajima: Acta Mater., 56 (2008), 2856. https://doi. org/10.1016/j.actamat.2008.02.017

8) W. Kaita, K. Hagihara, L. A. Rocha and T. Nakano: Scr. Mater., 142 (2018), 111. https://doi.org/10.1016/j.scriptamat.2017.08.016

9) T. P. Gabb, J. Gayda and R. V. Miner: Metall. Trans. A, 17 (1986), 497. https://doi.org/10.1007/BF02643956

10) V. Sass, U. Glatzel and M. Feller-Kniepmeier: Acta Mater., 44 (1996), 1967. https://doi.org/10.1016/1359-6454(95)00315-0

11) K. Hagihara, T. Nakano, M. Suzuki, T. Ishimoto, Suyalatu and S.-H Sun: J. Alloy. Compd., 696 (2017), 67. https://doi.org/10.1016/j. jallcom.2016.11.191

12) M. Bayat, A. Thanki, S. Mohanty, A. Witvrouw, S. Yang, J. Thorborg, N. S. Tiedje and J. H. Hattel: Addit. Manuf., 30 (2019), 100835. https://doi.org/10.1016/j.addma.2019.100835

13) S.-H. Sun, T. Ishimoto, K. Hagihara, Y. Tsutsumi, T. Hanawa and T. Nakano: Scr. Mater., 159 (2019), 89. https://doi.org/10.1016/j. scriptamat.2018.09.017

14) P. C. Collins, D. A. Brice, P. Samimi, I. Ghamarian and H. L. Fraser: Annu. Rev. Mater. Res., 46 (2016), 63. https://doi.org/10.1146/ annurev-matsci-070115-031816

15) F. Yan, W. Xiong and E. J. Faierson: Materials, 10 (2017), 1260 https://doi.org/10.3390/ma10111260

16) J. N. Dupont: ASM Handbook, 6A: Welding Fundamentals and Processes, ed. by T. Lienert et al., ASM International, Materials Park, OH, (2011), 96.

17) S. Bontha, N. W. Klingbeil, P. A. Kobryn and H. L. Fraser: Mater. Sci. Eng. A, 513-514 (2009), 311. https://doi.org/10.1016/j. msea.2009.02.019

18) P. Promoppatum, S. C. Yao, P. C. Pistorius and A. D. Rollett: Engineering, 3 (2017), 685. https://doi.org/10.1016/J.ENG.2017.05.023
19) M. Todai, T. Nakano, T. Liu, H. Y. Yasuda, K. Hagihara, K. Cho, M. Ueda and M. Takeyama: Addit. Manuf., 13 (2017), 61. http://doi. org/10.1016/j.addma.2016.11.001

20) O. Andreau, I. Koutiri, P. Peyre, J. D. Penot, N. Saintier, E. Pessard, T. De Terris, C. Dupuy and T. Baudin: J. Mater. Process. Technol., 264 (2019), 21. https://doi.org/10.1016/j.jmatprotec.2018.08.049

21) C. Zhao, K. Fezzaa, R. W. Cunningham, H. Wen, F. De Carlo, L. Chen, A. D. Rollett and T. Sun: Sci. Rep., 7 (2017), 3602. https://doi. org/10.1038/s41598-017-03761-2

22) X. Wang, J. A. Muñiz-Lerma, M. Attarian Shandiz, O. Sanchez-Mata and M. Brochu: Mater. Sci. Eng. A, 766 (2019), 138395. https://doi. org/10.1016/j.msea.2019.138395

23) A. Parsapour, S. N. Khorasani and M. H. Fathi: J. Mater Sci. Technol., 28 (2012), 125. https://doi.org/10.1016/S1005-0302(12)60032-2

24) R. Huiskes, H. Weinans and B. V. Rietbergen: Clin. Orthop. Relat. Res., 274 (1992), 124. https://doi.org/10.1097/00003086-19920100000014

25) Y. Noyama, T. Miura, T. Ishimoto, T. Itaya, M. Niinomi and T. Nakano: Mater. Trans., 53 (2012), 565. https://doi.org/10.2320/ matertrans.M2011358

26) T. Nakano, K. Kaibara, Y. Tabata, N. Nagata, S. Enomoto, E. Marukawa and Y. Umakoshi: Bone, 31 (2002), 479. https://doi. org/10.1016/s8756-3282(02)00850-5

27) T. Ishimoto, T. Nakano, Y. Umakoshi, M. Yamamoto and Y. Tabata: J. Bone Miner. Res., 28 (2013), 1170. https://doi.org/10.1002/ jbmr. 1825

28) J. Wang, T. Ishimoto and T. Nakano: Calcif. Tissue Int., 100 (2017), 87. https://doi.org/10.1007/s00223-016-0200-0

29) S. Kuroshima, T. Nakano, T. Ishimoto, M. Sasaki, M. Inoue, M. Yasutake and T. Sawase: Acta Biomater., 48 (2017), 433. https://doi. org/10.1016/j.actbio.2016.11.021

30) M. Niinomi: Sci. Technol. Adv. Mater., 4 (2003), 445. https://doi. org/10.1016/j.stam.2003.09.002

31) F.-Q. Yang, H. Xue, L.-Y. Zhao, X.-R. Fang and H.-B. Zhang: Adv. Mater. Sci. Eng., 2019 (2019), 2468487. https://doi. org/10.1155/2019/2468487

32) S. Gorsse, C. Hutchinson, M. Gouné and R. Banerjee: Sci. Technol. Adv. Mater., 18 (2017), 584. https://doi.org/10.1080/14686996.2017. 1361305

33) C. Qiu, M. A. Kindi, A. S. Aladawi and I. A. Hatmi: Sci. Rep., 8 (2018), 7785. https://doi.org/10.1038/s41598-018-26136-7 